GRADIATION\&APPLICATIONS

ISSN 2466-4294 (online) | rad-journal.org

Vol. 2 | Issue 3 | pp. $158-163,2017$

doi: 10.21175/RadJ.2017.03.033

Original research paper

\title{
RADIATION DOSE-RATE EFFECTS ON SELECT BIOMARKERS IN A MOUSE TOTAL-BODY IRRADIATION MODEL
}

\author{
Sara R. Hegge* , Gregory L. King \\ Armed Forces Radiobiology Research Institute (AFRRI), \\ Uniformed Services University of the Health Sciences (USUHS), Bethesda, MD
}

\begin{abstract}
In the event of acute radiation exposure, absorbed dose may be unknown and biodosimetry tools are needed by first responders to properly triage patients. We evaluated two protein markers - FMS-related tyrosine kinase 3 ligand (Flt3L) and granulocyte colony-stimulating factor $(G-C S F)$ - that are known to be elevated after an acute radiation exposure, as well as total white blood cell (WBC) count changes pre- and post-irradiation at different dose-rates. Female B6D2F1 mice were divided into one sham-irradiated control group and four total-body irradiated groups. Experimental groups received a total dose of 8 Gy of ${ }^{60} \mathrm{Co}$ gamma photon irradiation at four dose-rates:

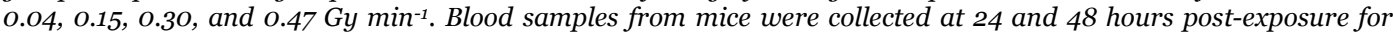
WBC and protein biomarkers (Flt3L and G-CSF). Flt3L values at all dose-rates except 0.15 were significantly elevated

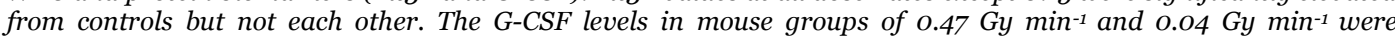

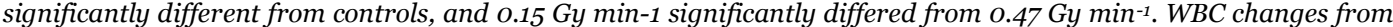
baseline showed that all experimental groups were significantly lower than controls, and additionally the o.04 Gy

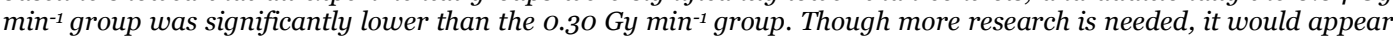
that at the fixed dose, dose-rates, and time points chosen herein may not be particularly strong or show predictable differences in the selected biomarker expression levels.
\end{abstract}

Key words: Bioassay; biological indicators; dose-rate; total- body irradiation

\section{INTRODUCTION}

Numerous people (civilian and military) could be exposed to ionizing radiation after a terrorist radiological attack, a radiation or nuclear accident, or a combat detonation. In such scenarios, triage tools are needed by first-responders to identify victims exposed to life-threatening doses of radiation, as well as discriminate between those not exposed and those exposed but expected to survive. Such casualties could occur in response to an acute or prompt irradiation dose as well as a protracted one. Triage tools are available for both these scenarios [1], but under masscasualty conditions, it will be important to assess radiation dose and injury as quickly as possible. The aforementioned authors state that proteomics may have an impact on biodosimetry and that proteomics may prove more informative (i.e. "protein changes may be more relevant, longer lasting or more indicative of long-term cellular damage correlating with biological impact and risk"). It is the purpose of this study to evaluate two identified protein markers (known to be elevated after an acute radiation dose) for their utility after varied radiation dose-rates. Complete Blood Count (CBC) values are also compared under these conditions. Multiple biomarker measures together may all provide better discernment among irradiation doses

*sara.r.hegge.mil@mail.mil and the degree of acute radiation syndrome (ARS) sequelae expected [2-5].

There are several candidate biomarkers available for acute radiation exposure and two of the most promising are fms-like tyrosine kinase receptor-3 (Flt3L) and granulocyte colony stimulating factor (G-CSF) [6-8]. In addition, CBC values such as white blood cell count can provide further information on the degree of damage in response to irradiation. These three biomarkers are evaluated.

Flt3L is a class III receptor tyrosine kinase that holds promise as a therapeutic target in hematological malignancies [9] and acts as a growth factor to increase the number of immune cells by activating hematopoietic progenitor cells, mobilizing them and also mobilizing stem cells. Bertho et al. [10] found that plasma Flt3L concentrations in non-human primates (NHP) increased as early as day 2 post-irradiation and by day 5 , the levels correlated with both radiation dose and severity of radiation-induced aplasia. The peak of the Flt3L concentration appeared before the nadir of the neutrophil count and subsequent decreases in Flt3L concentration correlated with hematologic recovery. The same group demonstrated that Flt3L plasma concentrations reflect post-irradiation bone marrow function in two mouse strains, the BALB/c and NOD-SCID [11], and in the BALB/c strain also correlated with the severity of aplasia [12]. This group 
S. R. Hegge, G. L. King, Radiation dose-rate effects on select biomarkers..., Rad. Applic., 2017, 2, 3, 158-163

also showed that the overall amount of Flt3L correlated directly with the cumulative radiation dose and the proportion of irradiated bone marrow in humans during a fractionated-radiation therapy trial [13]. The Flt3L assay was also used to demonstrate the severity of the bone marrow aplasia after two different radiation accidents $[14,15]$. Thus, for biodosimetry after acute irradiation exposure, Flt3L has been validated as a marker in mice, NHPs and humans.

A second potential biomarker is G-CSF, a colonystimulating factor produced by numerous tissues to stimulate the bone marrow to produce granulocytes and stem cells, then stimulates the bone marrow to release the granulocytes into the bloodstream and also stimulates the survival, proliferation, differentiation and function of neutrophil precursors and mature neutrophils. It is commonly used off-label in clinics after radiological incidents or accidents $[16,17]$. It is the therapeutic standard for irradiation injuries below the LD50 [16], is standard treatment for the Acute Radiation Syndrome in the US [18], and is stored in the Strategic National Stockpile of the Centers for Disease Control and the World Health Organization. Of importance is that it has been shown that G-CSF is released in mouse and NHP radiation models in a dose-dependent manner in response to ionizing radiation $[3,19]$. This has been substantiated by Desai et al. [20] who found G-CSF (and other cytokines) to be secreted into the conditioned media of five different human tumor cell lines 48 hours after irradiation. Although a full dose-response curve for irradiation was not generated, 6 Gy irradiation evoked a significantly greater release of G-CSF than did 2 Gy. Thus this relatively new protein biomarker for radiation injury should be assessed for the effects of dose-rate on its production.

Diminished $\mathrm{CBC}$ values are well documented to occur within 24-48 hours after acute irradiations in a dose-dependent manner. There is no significant doserate effect of gamma irradiation on circulating erythrocytes and leukocytes when comparing high and low dose-rates [21-24], although the latter low doserates are far lower than the ones investigated herein. However, there is a dose-rate effect on thrombocytes [23], and low dose-rate irradiation significantly decreases leukocytes, erythrocytes and WBCs in a dosedependent fashion [21-23]. Radiation accidents and studies related to the space program provide the most information regarding the effect of low dose-rate irradiation on $\mathrm{CBC}$ values. For example, WBC and platelet values were evaluated for three victims accidently exposed to a ${ }^{60}$ Co source for $9.3-20$ hours and the physical doses were determined to be between 2.4 and 6.1 Gy [17]. Both WBC and platelet values began falling almost immediately after exposure and the lowest counts occurred three weeks past the accident (despite receiving almost immediate and prolonged medical treatment). In another accidental exposure, five victims were exposed to ${ }^{60} \mathrm{Co}$ for $12-14 \mathrm{hr}$ daily for approximately a week [26]. There was severe neutropenia in four of the victims and thrombocytopenia ranged from severe to low normal. Chromosomal analysis yielded radiation dose estimates ranging between 0.6-3.1 Gy.
The National Aeronautic and Space Administration (NASA) has long been interested in the effects of low dose-rate irradiation, particularly from radiation qualities that may be encountered in space. Gammairradiation is often compared with high LET irradiations in these studies. It has been shown that there is no significant dose-rate effect of gamma irradiation on circulating erythrocytes and leukocytes when comparing high and low dose-rates [21-23], although the low dose-rates are lower than the ones used here. However, there is a dose-rate effect on thrombocytes [23] and low dose-rate irradiation significantly decreases leukocytes, erythrocytes and WBCs in a dose-dependent fashion [21-23]. In the study by Gridley et al.[23], the radiation doses were $0.5,1.5$ and 3 Gy at $1 \mathrm{cGy} \mathrm{min}^{-1}$. In the Pecaut et al. [22] study, the irradiation doses were again $0.5,1.5$ and 3 Gy and the dose rates compared were $1 \mathrm{cGy} \mathrm{min}^{-1} \mathrm{vs}$.

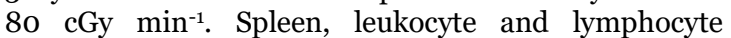
changes were altered in a dose-dependent manner, but not in response to dose rate. In the Maks et al. [21] study, the irradiation doses ranged from $0.5-2$ Gy at $0.5 \mathrm{~Gy} \mathrm{~min}^{-1}$ vs. $0.5 \mathrm{~Gy} \mathrm{hr}^{-1}$.

Although the protein biomarkers FLt3L and G-CSF appear to be valuable in ascertaining the radiation response to acute irradiation doses, it is unknown whether they will be elevated in a similar manner and/or with a similar time-course after substantially lowered radiation dose-rates of equivalent cumulative dose in vivo. Our hypothesis was that there are detectable differences amongst dose-rate groups for the biomarkers chosen. The utility for elucidating these differences could have practical implications for dosimetry with respect to dose-rate, compared to total dose.

\section{MATERIALS AND METHODS}

\subsection{Animals}

Eighty four female B6D2F1 mice (Mus musculus) 10-20 wk old (approximately 22-26 g) were used for these studies. Mice were housed under conventional conditions in microisolator filter-top cages in a facility fully accredited by the Association for Assessment and Accreditation of Laboratory Animal Care (AAALAC) International and treated in accordance with principles outlined in the Guide for the Care and Use of Laboratory Animals of the Institute of Laboratory Animal Resources, National Research Council. Animal rooms were provide with $10-15$ air changes h-1 of $100 \%$ fresh conditioned air and maintained at $22( \pm 2){ }^{\circ} \mathrm{C}$ and a relative humidity of $50( \pm 20) \%$. Animals remained on 12:12-h light:dark cycles and received food (Rodent Diet \#8604, Harlan Teklad, Madison, WI) and water acidified with $\mathrm{HCl}$ to a $\mathrm{pH}$ of 2.5-2.8) ad libitum. Mice were acclimated for 1 week before preirradiation CBC blood collection. Mice tails were tattooed for individual identification during acclimation. Animal manipulations and research were conducted under an approved IACUC protocol. 
S. R. Hegge, G. L. King, Radiation dose-rate effects on select biomarkers..., Rad. Applic., 2017, 2, 3, 158-163

\subsection{Exposure of animals to $\gamma$-radiation and blood collection}

CBCs were obtained from all mice approximately 3 weeks prior to irradiation to compare to postirradiation values. The facial vein venipuncture technique was used, under isoflurane anesthesia, to acquire approximately $50 \mu \mathrm{L}$ of blood in an EDTA tube. All mice recovered from anesthesia and venipuncture without complications. A CBC analyzer was used that was able to process this small amount of blood ("scil Vet abc" Hematology Analyzer, "scil animal care company", Gurnee, IL).

For the irradiations, AFRRI's ${ }^{60}$ Co facility was used and the mice were housed in Plexiglas partition chambers that fit into micro-environment cages (4 partitions per cage with dimensions of each chamber at 2.5 " wide x 4 " deep x $61 / 5$ " tall; Figure 1) and allowed to move freely about with access to food and water during the irradiation exposures. Due to the partitioning, the mice were irradiated unilaterally with Cobalt-6o gamma photons, and could not shield one another. The longer exposure times (up to 215 minutes) required for the low dose-rate irradiations necessitated this unilateral setup, as using the smaller restraint boxes available for bilateral irradiation would have resulted in undesirable prolonged restraint. It was not possible to bilaterally-irradiate these cages due to the potential for shielding/scatter of irradiation from a second beam passing through the back side of the cage where water bottles and food were located.

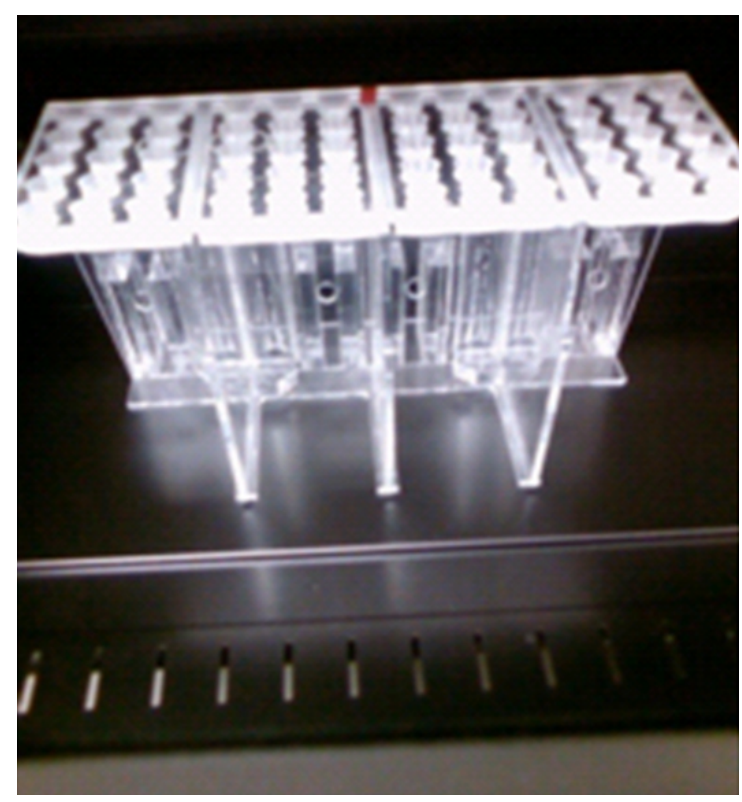

Figure 1. Plexiglas $®$ insert for mouse shoe-box cage that allows freedom of movement for mice as well as access to food and water during the irradiation procedure. The tubes for food and water are behind the open chambers. The insert dimensions are described in the text.

The ${ }^{60}$ Co irradiation dose was fixed at $8 \mathrm{~Gy}$ for all groups as a mean LD50/30 for this strain has recently been described as $9.39 \mathrm{~Gy}$ in response to bilateral irradiation [25]. The 8.0 Gy dose was chosen as it was close to the reported $\mathrm{LD}_{50} / 30$, such that it was likely to induce hematologic changes, however unlikely to produce mortality from ARS at 48 hours postirradiation. There were 4 irradiated groups of 16 mice per group at the following requested dose-rates: 0.05, $0.2,0.4$, and $0.6 \mathrm{~Gy} \mathrm{~min}^{-1}$ (equivalent to an exposure time range of 17-215 min). However, due to radioactive decay from the date of dosimetry mapping, actual dose rates delivered were 0.04, 0.15, 0.30, and $0.47 \mathrm{~Gy} \mathrm{~min}^{-1}$ (rounded to the hundredth). Additionally, the actual lengths of time were adjusted by the facility dosimetry team to account for radioactive decay of the radiation source (Table 1 ). The originally requested dose rate of $0.6 \mathrm{~Gy} \mathrm{~min} \mathrm{~m}^{-1}$ is a lynch-pin dose rate for the varied dose rates because previous and ongoing work at AFRRI on biomarkers has been done at this dose rate.

Table 1 . Dose-rates by group

\begin{tabular}{|c|c|c|c|c|c|}
\hline Group & $\mathrm{A}$ & $\mathrm{B}$ & $\mathrm{C}$ & $\bar{D}$ & Controls \\
\hline Dose Rate (G & 0.47 & 0.30 & 0.15 & 0.04 & 0 \\
\hline Total min. irradiation & $13 \cdot 3$ & 26 & 53 & 160 & 0 \\
\hline
\end{tabular}

Twenty-four hours after irradiation half the mice from each group were deeply anesthetized under isoflurane anesthesia for cardiac puncture to obtain blood for CBCs. Forty-eight hours after irradiation, the other half of the mice from each group were deeply anesthetized via the same procedure to obtain blood for CBC, Flt3L and G-CSF. For each time point, after the maximum amount of blood was withdrawn (up to approximately $0.9 \mathrm{~mL}$ ) and the mice were humanely euthanized by exsanguination under general anesthesia followed by cervical dislocation. Approximately 50-100 $\mu \mathrm{L}$ was placed in an EDTA tube for CBC analysis and the remainder, approximately $100-800 \mu \mathrm{L}$, placed in a no-additive tube for serum biomarker analysis. CBCs were measured using the same veterinary hematology analyzer utilized for the pre-irradiation collections. The blood collected in no-additive tubes was allowed to clot for 2 hours, then centrifuged at $2000 \mathrm{~g}$ for $20 \mathrm{~min}$, and serum was aliquoted into 2 tubes per sample and stored at $-20^{\circ} \mathrm{C}$ for protein bioassays.

Samples for G-CSF and Flt3L measurements were packed on dry ice, shipped overnight, and analyzed by R\&D Systems' Biomarker Testing Service (Minneapolis, MN), using the R\&D Systems' Mouse G-CSF Quantikine Enzyme-linked Immunosorbent Assay (ELISA) kit and R\&D Systems' Mouse/Rat Flt-3 Ligand Quantikine ELISA kit.

\subsection{Statistical Analysis}

Statistical software (SPSS 22) was used for statistical data analysis. Assumptions of a traditional ANOVA were not met (i.e. normal distribution and equal variances assumed) and therefore a nonparametric test was used. The Kruskal-Wallis test was used to compare distributions across groups, and Dunn's post-hoc test was used for all pairwise comparisons among groups. Significance levels were set at $p<0.05$. 
S. R. Hegge, G. L. King, Radiation dose-rate effects on select biomarkers..., Rad. Applic., 2017, 2, 3, 158-163

\section{RESULTS}

\subsection{Flt3L}

Control group levels of Flt3L had a median and interquartile range of 340.6 and $36.9 \mathrm{pg} \mathrm{mL}-1$ (Figure 2). These are slightly higher but comparable to published control levels in $\mathrm{CD} 2 \mathrm{~F} 1$ male mice [3]. The Kruskal-Wallis test gave an asymptotic significance value of $p<0.001$ thus rejecting the null hypothesis that the distribution of Flt3 values was the same across groups.

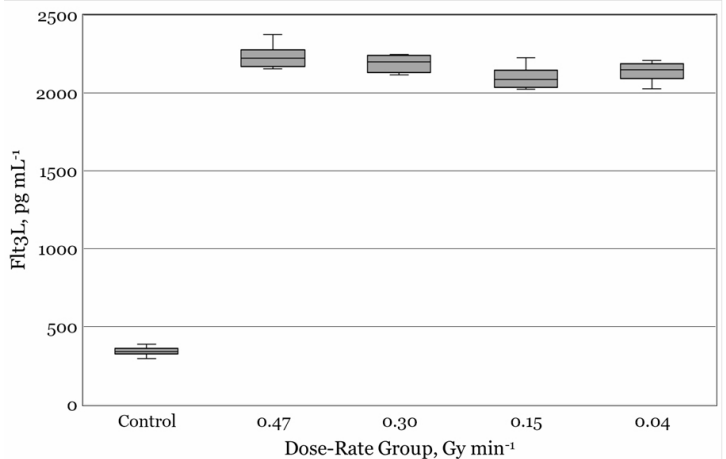

Figure 2. Flt3L ligand differences as determined for independent samples obtained 48 hours post-irradiation by the Kruskal-Wallis Test. Total N $=38 ; p<0.001$. Dark lines in box plots represent median values, boxes extend from the $25^{\text {th }}$ to $75^{\text {th }}$ percentile, and whiskers extend to the minimum and maximum values.

The Flt3L values for the experimental dose-rate groups of $0.47 \mathrm{~Gy} \mathrm{~min}^{-1}, 0.30 \mathrm{~Gy} \mathrm{~min}^{-1}$ and $0.04 \mathrm{~Gy} \mathrm{~min}^{-1}$ differed significantly from control when evaluated by the post-hoc tests using Dunn's adjustment for multiple comparisons.

\section{2. $G-C S F$}

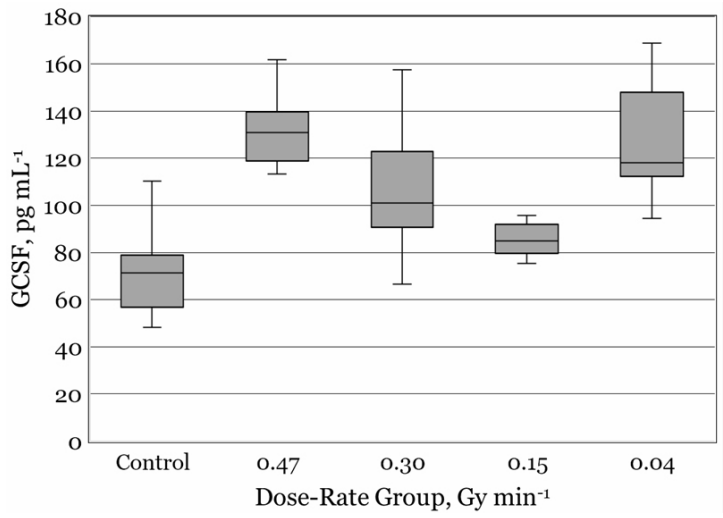

Figure 3. G-CSF ligand differences for independent samples obtained 48 hours post-irradiation as determined by the Kruskal-Wallis. Total $\mathrm{N}=38, p<0.001$. Dark lines in box plots represent median values, boxes extend from the $25^{\text {th }}$ to $75^{\text {th }}$ percentile, and whiskers extend to the minimum and maximum values.
Control group levels of G-CSF had a median and interquartile range of 71.3 and $22.2 \mathrm{pg} \mathrm{mL}-1$ (Figure 3) similar to published sham-irradiated levels in the CD2F1 strain [3]. Again, the Independent-Samples Kruskal-Wallis Test was used to determine if the distribution of G-CSF was the same across categories of the Group; this null hypothesis was rejected with an asymptotic significance value of $p<0.001$. The 0.47 Gy min $^{-1}$ and $0.04 \mathrm{~Gy} \mathrm{~min}^{-1}$ values differed significantly from control values and the 0.47 Gy min-1 dose-rate values differed from the values for the $0.15 \mathrm{~Gy} \mathrm{~min}^{-1}$ values (Figure 3). The medians of these groups are not likely to be equal.

\section{3. $W B C$}

For the WBC the distribution of the percent change was not the same across categories as tested by the Independent Samples Kruskal-Wallis Test $(p<0.001)$. For these tests both the 24 and $48 \mathrm{~h}$ post-irradiation results were pooled. When the two post-irradiation time-points were compared for each dose-rate, there were no differences between their median values (data not shown). The control values significantly differed from all dose-rate group values, and among experimental groups only the $0.30 \mathrm{~Gy} \mathrm{~min}^{-1}$ dose-rate values differed significantly from the $0.04 \mathrm{~Gy} \mathrm{~min}^{-1}$ values (Figure 4).

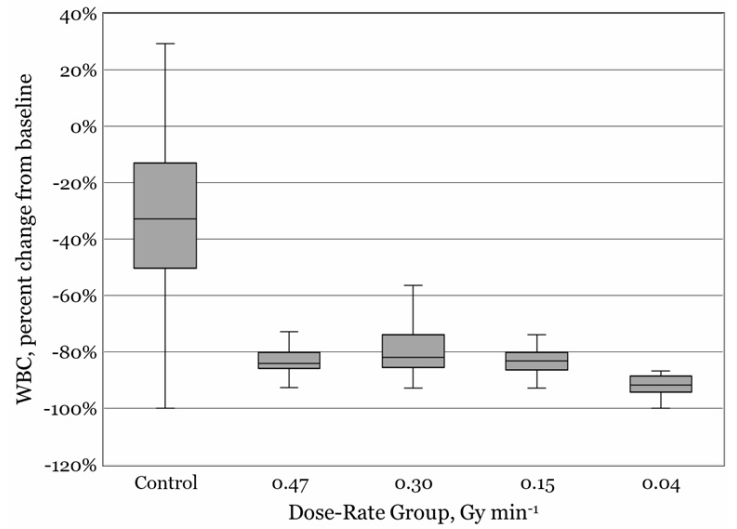

Figure 4. Percent change from baseline of WBC counts as determined for independent samples by the Kruskal-Wallis Test for ordered alternatives. Twenty-four and $48 \mathrm{~h} \mathrm{WBC}$ values are pooled together. Total $\mathrm{N}=78, \mathrm{p}<0.001$. Dark lines in box plots represent median values, boxes extend from the $25^{\text {th }}$ to $75^{\text {th }}$ percentile, and whiskers extend to the minimum and maximum values.

\subsection{Limitations}

Sample volumes from all mice for all tests were incomplete due to insufficient sample volume collected. Additionally, some samples were only able to be run singly rather than as duplicates in the ELISA plates. $\mathrm{CBC}$ results from 4 mice were excluded due to insufficient sample volume collected. Note that Flt3L and G-CSF were from the same mice but some had insufficient sample volume to run both tests resulting in sample sizes of less than 8 mice per group.

After irradiation, all CBC samples had absolute WBC counts but most values were too low to give 
S. R. Hegge, G. L. King, Radiation dose-rate effects on select biomarkers..., Rad. Applic., 2017, 2, 3, 158-163

differential breakdowns of the components (lymphocytes, monocytes, and granulocytes). Because WBC values (in particular the lymphocyte count) are known to drop dramatically post-irradiation, it was appropriate to measure the changes in absolute WBC counts. To observe the changes post-irradiation, the percent change from baseline (pre-irradiation) count was calculated. This was calculated by subtracting the pre-irradiation count from the post-irradiation count, and taking that number divided by the pre-irradiation count.

Both ELISA tests were only validated for results up to the highest standard of $2000 \mathrm{pg} \mathrm{mL}-1$. Therefore, even though some test results gave values over this amount, they could not be reported more accurately without repeating the assay with diluent and multiplying by the dilution factor. As the minimum required serum sample in duplicate was $100 \mu \mathrm{L}$, there was insufficient sample volume to repeat the assays. Additionally, some samples only had enough volume to run singly rather than in duplicate.

\section{Discussion}

From what we could evaluate, results from the ELISA tests did not appear to demonstrate much of a dose-rate effect. Overall the tests showed that there were at least some significant differences between the experimental groups and the control group, but not many significant differences between experimental groups.

Bertho and Roy have shown the utility of using Flt3L measurements to assess radiation accident victims in Dakar [14]. In this accident, an iridium-192 source was stored close to a work place for approximately 8 weeks potentially exposing 63 victims. The biological evaluation took place at least 4 weeks after the end of irradiation. In this study plasma FLt3L was evaluated in addition to $\mathrm{CBC}$ alterations and a scoring system was developed based on these hematological parameters and compared with a scoring system for chromosomal aberrations. The two scoring systems were strongly correlated and there were 10 patients identified with a final global score (for $\mathrm{CBC}+$ FLt3L) greater than 3. For the patients with scores $\geq 4$ the authors concluded (using this method) that anyone with such scores would be at risk for the ARS and should be placed under medical supervision. Their data also suggested that the FLt3L concentration analysis plus CBC analysis may serve as a method for rapid evaluation of radiation injury. This analysis was also applied to another accident victim [15]. The long-term results from a fractionated human radiation study [13] further support the notion that at least FLt3L may respond to radiation at a protracted dose rate. These two studies clearly involve radiation exposures in which the repair and recovery processes would continue despite the irradiations, unlike with this study.

Our data may indicate that in dose-rate dosimetry, WBC counts may be the most helpful and practical indicator of exposure, potentially at dose-rates $0.04 \mathrm{~Gy}$ $\min ^{-1}$ and below, but has a somewhat paradoxical effect. Although not considered in our original hypothesis, we would have expected the lowest doserate to be affected the least by protracted radiation because of DNA repair mechanisms, but our data showed the opposite.

Overall, the dose-rates in this study did not show a strong or predictable pattern when compared to each other. However, our study had multiple limitations, and more research is needed, especially at lower doserates, to determine if a dose-rate effect truly exists when measuring biomarker levels. The selected biomarkers remain important tools in estimating total dose exposure, though utility for dose-rate estimation remains unclear.

Acknowledgement: This project was funded by intramural grant $R A B \# 3333214$. The authors would like to thank Vilmar Villa for assistance with sample aliquots; Dr. Cara Olsen and Dr. Adarsh Ramakumar for biostatistical input; Dr. Natalia Ossetrova for biomarker consultation; Dr. Alexandra Miller for use of hematology analyzer; and VSD technicians for assistance with blood draws.

Disclaimer: The content of this publication is the sole responsibility of the author(s) and does not necessarily reflect the views or policies of the Armed Forces Radiobiology Research Institute (AFRRI), Uniformed Services University of the Health Sciences (USUHS), the Department of Defense (DoD), or the Departments of the Army, Navy, or Air Force. Mention of trade names, commercial products, or organizations does not imply endorsement by the US Government.

\section{REFERENCES}

1. S. A. Amundson et al., "Biological indicators for the identification of ionizing radiation exposure in humans," Expert Rev Mol Diagn, vol. 1, no. 2, pp. 211 219, Jul. 2001.

DOI: 10.1586/14737159.1.2.211

PMid: 11901816

2. N. I. Ossetrova and W.F. Blakely, "Multiple bloodproteins approach for early-response exposure assessment using an in vivo murine radiation model," Int. J. Radiat. Biol., vol. 85. no. 10, pp. 837 - 850, Oct. 2009.

DOI: $10.1080 / 09553000903154799$ PMid: 19863200

3. N. I. Ossetrova et al., "Early-response biomarkers for assessment of radiation exposure in a mouse total-body irradiation model," Health Phys., vol. 106, no. 6, pp. 772 - 786, Jun. 2014.

DOI: 10.1097/HP.0000000000000094 PMid: 24776912

4. N. I. Ossetrova et al., "Acute Radiation Syndrome Severity Score System in Mouse Total-Body Irradiation Model," Health Phys., vol. 111, no. 2, pp. 134 - 144 Aug. 2016.

DOI: $10.1097 /$ HP.ooooooooooooo499

PMid: 27356057

5. N. I. Ossetrova et al., "Combined approach of hematological biomarkers and plasma protein SAA for improvement of radiation dose assessment triage in biodosimetry applications," Health Phys., vol. 98, no. 2, pp. 204 - 208, Feb. 2010.

DOI: 10.1097/HP.obo13e3181abaabf

PMid: 20065684 
S. R. Hegge, G. L. King, Radiation dose-rate effects on select biomarkers..., Rad. Applic., 2017, 2, 3, 158-163

6. P. G. Prasanna et al., "Synopsis of partial-body radiation diagnostic biomarkers and medical management of radiation injury workshop," Radiat. Res., vol. 173, no. 2, pp. 245 - 253, Feb. 2010. DOI: $10.1667 /$ RR1993.1 PMid: 20095857

7. T. Straume et al., "NASA Radiation Biomarker Workshop, September 27-28, 2007," Radiat. Res., vol. 170, no. 3, pp. 393 - 405, Sep. 2008. DOI: $10.1667 / \mathrm{RR} 1382.1$ PMid: 18763867

8. C. Gabay and I. Kushner, "Acute-phase proteins and other systemic responses to inflammation," N. Engl. J. Med., vol. 340, no. 6, pp. 448 - 454, Feb, 1999. DOI: 10.1056/NEJM199902113400607 PMid: 9971870

9. H. Youssoufian et al., "Targeting FMS-related tyrosine kinase receptor 3 with the human immunoglobulin G1 monoclonal antibody IMC-EB10," Cancer, vol. 116, no. suppl. 4, pp. 1013 - 1017, Feb. 2010.

DOI: $10.1002 /$ cncr.24787 PMid: 20127944

10. J. M. Bertho, et al., "Level of Flt3-ligand in plasma: a possible new bio-indicator for radiation-induced aplasia," Int. J. Radiat. Biol., vol. 77, no. 6, pp. 703 712, Jun. 2001.

DOI: $10.1080 / 09553000110043711$ PMid: 11403710

11. M. Prat et al., "Radiation-induced increase in plasma Flt3 ligand concentration in mice: evidence for the implication of several cell types," Radiat. Res., vol. 163, no. 4, pp. 408 - 417, Apr. 2005.

DOI: $10.1667 / \mathrm{RR} 3340$ PMid: 15799697

12. M. Prat et al., "Use of flt3 ligand to evaluate residual hematopoiesis after heterogeneous irradiation in mice," Radiat. Res., vol. 166, no. 3, pp. 504 - 511, Sep. 2006. DOI: $10.1667 /$ RRo568.1 PMid: 16953669

13. A. Huchet et al., "Plasma Flt-3 ligand concentration correlated with radiation-induced bone marrow damage during local fractionated radiotherapy," Int. J. Radiat. Oncol. Biol. Phys, vol. 57, no. 2, pp. 508 - 515, Oct. 2003.

DOI: 10.1016/So360-3016(03)00584-4

14. J. M. Bertho et al., "Initial evaluation and follow-up of acute radiation syndrome in two patients from the Dakar accident,” Biomarkers, vol. 14, no. 2, pp. 94 102, 2009.

DOI: $10.1080 / 13547500902773904$ PMid: 19330587

15. J. M. Bertho, et al., "New biological indicators to evaluate and monitor radiation-induced damage: an accident case report," Radiat. Res., vol. 169, no. 5, pp. 543 - 550, May 2008.

DOI: $10.1667 / R R 1259.1$ PMid: 18439044

16. M. Drouet and F. Hérodin, "Radiation victim management and the haematologist in the future: time to revisit therapeutic guidelines?" Int. J. Radiat. Biol., vol. 86, no. 8, pp. $636-648$, Jul. 2010.

DOI: $10.3109 / 09553001003789604$

PMid: 20597842
17. Q. Liu et al., "Clinical report of three cases of acute radiation sickness from a (60)Co radiation accident in Henan Province in China," J. Radiat. Res., vol. 49, no. 1, pp. 63 - 69, Jan. 2008.

DOI: 10.1269/jrr.07071

18. L. Heslet, C. Bay and S. Nepper-Christensen, "Acute radiation syndrome (ARS) - treatment of the reduced host defense," Int. J. Gen. Med., vol. 5, pp. 105 - 115, 2012.

DOI: 10.2147/IJGM.S22177

PMid: 22319248

PMCid: PMC 3273373

19. N. I. Ossetrova et al., "Non-human Primate Total-body Irradiation Model with Limited and Full Medical Supportive Care Including Filgrastim for Biodosimetry and Injury Assessment," Radiat. Prot. Dosimetry, vol. 172, no. 1-3, pp. 174 - 191, Dec. 2016.

DOI: $10.1093 / \mathrm{rpd} /$ ncw176 PMid: 27473690

20. S. Desai et al., "Cytokine profile of conditioned medium from human tumor cell lines after acute and fractionated doses of gamma radiation and its effect on survival of bystander tumor cells," Cytokine, vol. 61, no. 1, pp. 54 - 62, Jan. 2013.

DOI: 10.1016/j.cyto.2012.08.022 PMid: 23022376

21. C. J. Maks et al., "Analysis of white blood cell counts in mice after gamma- or proton-radiation exposure," Radiat. Res., vol. 176, no. 2, pp. 170 - 176, Aug. 2011.

DOI: $10.1667 / R R 2413.1$

PMid: 21476859 PMCid: PMC3575683

22. M. J. Pecaut, G. A. Nelson and D. S. Gridley, "Dose and dose rate effects of whole-body gamma-irradiation: I. Lymphocytes and lymphoid organs," In Vivo, vol. 15, no. 3, pp. 195 - 208, May-Jun. 2001. PMid: 11491014

23. D. S. Gridley et al., "Dose and dose rate effects of wholebody gamma-irradiation: II. Hematological variables and cytokines," In Vivo, vol. 15, no. 3, pp. 209 - 216 , May-Jun. 2001. PMid: 11491015

24. D. S. Gridley et al., "Low dose, low dose rate photon radiation modifies leukocyte distribution and gene expression in $\mathrm{CD} 4(+) \mathrm{T}$ cells," J. Radiat. Res., vol. 50, no. 2, pp. 139 - 150, Mar. 2009 DOI: $10.1269 /$ jrr.08095

25. J. G. Kiang et al., "Wound trauma increases radiationinduced mortality by activation of iNOS pathway and elevation of cytokine concentrations and bacterial infection," Radiat. Res., vol. 173, no. 3, pp. 319 - 332, Mar. 2010.

DOI: $10.1667 / R R 1892.1$ PMid: 20199217

26. A. B. Dey et al., "Radiation accident at Mayapuri scrap market, Delhi, 2010," Radiat. Prot. Dosimetry, vol. 151, no. 4, pp. 645 - 651, Oct. 2012.

DOI: $10.1093 / \mathrm{rpd} / \mathrm{ncs} 162$

PMid: 22914329 